Pacific Journal of Mathematics

A MULTILINEAR GENERATING FUNCTION FOR THE

H. M. (Hart Moham) Srivastava 


\section{A MULTILINEAR GENERATING FUNCTION FOR THE KONHAUSER SETS OF BIORTHOGONAL POLYNOMIALS SUGGESTED BY THE LAGUERRE POLYNOMIALS}

\section{H. M. SRivastava}

The polynomial sets $\left\{Y_{n}^{\alpha}(x ; k)\right\}$ and $\left\{Z_{n}^{\alpha}(x ; k)\right\}$, discussed by Joseph D. E. Konhauser, are biorthogonal over the interval $(0, \infty)$ with respect to the weight function $x^{\alpha} e^{-x}$, where $\alpha>-1$ and $k$ is a positive integer. The object of the present note is to develop a fairly elementary method of proving a general multilinear generating function which, upon suitable specializations, yields a number of interesting results including, for example, a multivariable hypergeometric generating function for the multiple sum:

$$
\begin{array}{r}
\sum_{n_{1}, \ldots, n_{r}=0}^{\infty}\left(m+n_{1}+\cdots+n_{r}\right) ! Y_{m+n_{1}+\cdots+n_{r}}^{\alpha}(x ; k) \\
\cdot \prod_{i=1}^{r}\left\{\frac{Z_{n_{i}}^{\beta_{i}}\left(y_{i} ; s_{i}\right) u_{i}^{n_{i}}}{\left(1+\beta_{i}\right)_{s_{i} n_{i}}}\right\},
\end{array}
$$

involving the Konhauser biorthogonal polynomials; here, by definition,

$$
\alpha>-1 ; \quad \beta_{i}>-1 ; \quad k, s_{i}=1,2,3, \ldots ; \quad \forall i \in\{1, \ldots, r\} .
$$

1. Introduction. Joseph D. E. Konhauser ([5]; see also [4]) introduced two interesting classes of polynomials: $Y_{n}^{\alpha}(x ; k)$ a polynomial in $x$, and $Z_{n}^{\alpha}(x ; k)$ a polynomial in $x^{k}, \alpha>-1$ and $k=1,2,3, \ldots$ For $k=1$, these polynomials reduce to the classical Laguerre polynomials $L_{n}^{(\alpha)}(x)$, and for $k=2$ they were encountered earlier by Spencer and Fano [8] in the study of the penetration of gamma rays through matter and were discussed subsequently by Preiser [7]. Also [5, p. 303]

$$
\begin{aligned}
& \int_{0}^{\infty} x^{\alpha} e^{-x} Y_{m}^{\alpha}(x ; k) Z_{n}^{\alpha}(x ; k) d x \\
&=\frac{\Gamma(k n+\alpha+1)}{n !} \delta_{m n}, \quad \forall m, n \in\{0,1,2, \ldots\},
\end{aligned}
$$


so that the Konhauser polynomial sets $\left\{Y_{n}^{\alpha}(x ; k)\right\}$ and $\left\{Z_{n}^{\alpha}(x ; k)\right\}$ are biorthogonal over the interval $(0, \infty)$ with respect to the weight function $x^{\alpha} e^{-x}$, where $\alpha>-1, k$ is a positive integer, and $\delta_{m n}$ is the Kronecker delta.

The following explicit expression for the polynomials $Z_{n}^{\alpha}(x ; k)$ was given by Konhauser [5, p. 304, Eq. (5)]:

$$
Z_{n}^{\alpha}(x ; k)=\frac{\Gamma(k n+\alpha+1)}{n !} \sum_{j=0}^{n}(-1)^{j}\left(\begin{array}{l}
n \\
j
\end{array}\right) \frac{x^{k j}}{\Gamma(k j+\alpha+1)} .
$$

Subsequently, Carlitz pointed out that [2, p. 427, Eq. (9)]

$$
Y_{n}^{\alpha}(x ; k)=\frac{1}{n !} \sum_{j=0}^{n} \frac{x^{j}}{j !} \sum_{l=0}^{j}(-1)^{l}\left(\begin{array}{l}
j \\
l
\end{array}\right)\left(\frac{l+\alpha+1}{k}\right)_{n},
$$

where $(\lambda)_{n}=\Gamma(\lambda+n) / \Gamma(\lambda)$.

In a recent paper [10] we derived various properties of (for example) the Konhauser biorthogonal polynomials $Y_{n}^{\alpha}(x ; k)$ by suitably specializing those of the Srivastava-Singhal polynomials $G_{n}^{(\alpha)}(x, h, p, k)$ which are defined by the generalized Rodrigues formula [14, p. 75, Eq. (1.3)]

$$
\begin{aligned}
G_{n}^{(\alpha)}(x, h, p, k)= & \frac{x^{-k n-\alpha} \exp \left(p x^{h}\right)}{n !} \\
& \cdot\left(x^{k+1} D_{x}\right)^{n}\left\{x^{\alpha} \exp \left(-p x^{h}\right)\right\}, \quad D_{x}=\frac{d}{d x},
\end{aligned}
$$

and given explicitly by [14, p. 77, Eq. (2.1)]

$$
G_{n}^{(\alpha)}(x, h, p, k)=\frac{k^{n}}{n !} \sum_{j=0}^{n} \frac{\left(p x^{h}\right)^{j}}{j !} \sum_{l=0}^{j}(-1)^{l}\left(\begin{array}{l}
j \\
l
\end{array}\right)\left(\frac{h l+\alpha}{k}\right)_{n},
$$

where the parameters $\alpha, h, k$ and $p$ are unrestricted, in general. In fact, by comparing (5) with Carlitz's result (3), we at once deduce the known relationship [13, p. 315, Eq. (83)]

$$
Y_{n}^{\alpha}(x ; k)=k^{-n} G_{n}^{(\alpha+1)}(x, 1,1, k), \quad \alpha>-1 ; k=1,2,3, \ldots,
$$

which was of fundamental importance in our paper [10].

The object of the present note is first to give a rather elementary proof of a general multilinear generating function for the SrivastavaSinghal polynomials $G_{n}^{(\alpha)}(x, h, p, k)$. We then show how this multilinear generating function can be further generalized and applied to derive a 
number of interesting results including, for example, a multivariable hypergeometric generating function for the multiple sum $(*)$ involving the product of several Konhauser biorthogonal polynomials. Our main result is contained in the following

THEOREM. For a bounded multiple sequence $\left\{\Lambda\left(n_{1}, \ldots, n_{r}\right)\right\}$ of arbitrary complex numbers, let

$$
\begin{aligned}
& \mathscr{H}\left[n_{1}, \ldots, n_{r} ; y_{1}, \ldots, y_{r}\right] \\
&=\sum_{j_{1}=0}^{\left[n_{1} / m_{1}\right]} \cdots \sum_{j_{r}=0}^{\left[n_{r} / m_{r}\right]} \frac{\left(-n_{1}\right)_{m_{1} j_{1}}}{j_{1} !} \cdots \frac{\left(-n_{r}\right)_{m_{r} j_{r}}}{j_{r} !} \\
& \cdot \Lambda\left(j_{1}, \ldots, j_{r}\right) y_{1}^{j_{1}} \cdots y_{r}^{j_{r}},
\end{aligned}
$$

where $m_{1}, \ldots, m_{r}$ are positive integers. Also let $\Delta_{r}$ be defined by

$$
\Delta_{r}=1-\sum_{i=1}^{r} u_{i}, \quad r=1,2,3, \ldots
$$

Then, for every nonnegative integer $m$,

(9)

$$
\begin{aligned}
& \sum_{n_{1}, \ldots, n_{r}=0}^{\infty}\left(m+n_{1}+\cdots+n_{r}\right) ! G_{m+n_{1}+\cdots+n_{r}}^{(\alpha)}(x, h, p, k) \\
& \cdot \mathscr{H}\left[n_{1}, \ldots, n_{r} ; y_{1}, \ldots, y_{r}\right] \frac{\left(u_{1} / k\right)^{n_{1}}}{n_{1} !} \cdots \frac{\left(u_{r} / k\right)^{n_{r}}}{n_{r} !} \\
= & k^{m} \exp \left(p x^{h}\right) \Delta_{r}^{-m-\alpha / k} \\
& \cdot \sum_{n, n_{1}, \ldots, n_{r}=0}^{\infty}\left(\frac{h n+\alpha}{k}\right)_{m+m_{1} n_{1}+\cdots+m_{r} n_{r}}\left(\frac{1}{n !}\right) \Lambda\left(n_{1}, \ldots, n_{r}\right)\left(-\frac{p x^{h}}{\Delta_{r}^{h / k}}\right)^{n} \\
& \cdot \prod_{i=1}^{r}\left\{\frac{\left[\left(-u_{i} / \Delta_{r}\right)^{m_{t}} y_{i}\right]^{n_{t}}}{n_{i} !}\right\}, \quad k \neq 0,
\end{aligned}
$$

provided that the multiple series on the right-hand side of (9) has a meaning, and

$$
\left|u_{1}+\cdots+u_{r}\right|<1
$$

2. Proof of the theorem. For convenience, let $\Omega\left(u_{1}, \ldots, u_{r}\right)$ denote the left-hand side of (9), and set

$$
N=n_{1}+\cdots+n_{r} \text { and } J=m_{1} j_{1}+\cdots+m_{r} j_{r} .
$$


Applying the explicit representation (5) and the definition (7), we find that

$$
\begin{aligned}
\Omega\left(u_{1}, \ldots, u_{r}\right)= & k^{m} \sum_{n_{1}, \ldots, n_{r}=0}^{\infty} u_{1}^{n_{1}} \ldots u_{r}^{n_{r}} \\
& \cdot \sum_{j=0}^{m+N} \frac{\left(p x^{h}\right)^{j}}{j !} \sum_{l=0}^{j}(-1)^{l}\left(\begin{array}{l}
j \\
l
\end{array}\right)\left(\frac{h l+\alpha}{k}\right)_{m+N} \\
& \cdot \prod_{i=1}^{r}\left\{\sum_{j_{i}=0}^{\left[n_{l} / m_{i}\right]} \frac{\left[(-1)^{m_{l}} y_{i}\right]^{j_{l}}}{j_{i} !\left(n_{i}-m_{i} j_{i}\right) !}\right\} \Lambda\left(j_{1}, \ldots, j_{r}\right) \\
= & k^{m} \sum_{j_{1}, \ldots, j_{r}=0}^{\infty} \Lambda\left(j_{1}, \ldots, j_{r}\right) \prod_{i=1}^{r}\left\{\frac{\left[\left(-u_{i}\right)^{m_{l}} y_{i}\right]^{j_{l}}}{j_{i} !}\right\} \\
& \cdot \sum_{n_{1}, \ldots, n_{r}=0}^{\infty} \frac{u_{1}^{n_{1}}}{n_{1} !} \cdots \frac{u_{r}^{n_{r}}}{n_{r} !} \sum_{j=0}^{m+N+J} \frac{\left(p x^{h}\right)^{j}}{j !} \\
& \cdot \sum_{l=0}^{j}(-1)^{l}\left(\begin{array}{l}
j \\
l
\end{array}\right)\left(\frac{h l+\alpha}{k}\right)_{m+N+J}
\end{aligned}
$$

Now we appeal to the series identity [9, p. 4, Eq. (12)]

$$
\begin{aligned}
\sum_{n_{1}, \ldots, n_{r}=0}^{\infty} f\left(n_{1}+\cdots+n_{r}\right) \frac{u_{1}^{n_{1}}}{n_{1} !} \cdots \frac{u_{r}^{n_{r}}}{n_{r} !} \\
=\sum_{n=0}^{\infty} f(n) \frac{\left(u_{1}+\cdots+u_{r}\right)^{n}}{n !},
\end{aligned}
$$

and (12) becomes

$$
\begin{aligned}
\Omega\left(u_{1}, \ldots, u_{r}\right)= & k^{m} \sum_{n, j_{1}, \ldots, j_{r}=0}^{\infty} \frac{\left(u_{1}+\cdots+u_{r}\right)^{n}}{n !} \\
& \cdot \prod_{i=1}^{r}\left\{\frac{\left[\left(-u_{i}\right)^{m_{i}} y_{i}\right]^{j_{l}}}{j_{i} !}\right\}_{j=0}^{m+n+J} \frac{\left(p x^{h}\right)^{j}}{j !} \\
& \cdot \sum_{l=0}^{j}(-1)^{l}\left(\begin{array}{l}
j \\
l
\end{array}\right)\left(\frac{h l+\alpha}{k}\right)_{m+n+J},
\end{aligned}
$$

where $J$ is defined, as before, by (11). 
The innermost sum in (14) is the $j$ th difference of a polynomial of degree $m+n+J$ in $\alpha$; it is nil when $j>m+n+J$. Thus we have

$$
\begin{aligned}
& \sum_{j=0}^{m+n+J} \frac{\left(p x^{h}\right)^{j}}{j !} \sum_{l=0}^{j}(-1)^{l}\left(\begin{array}{l}
j \\
l
\end{array}\right)\left(\frac{h l+\alpha}{k}\right)_{m+n+J} \\
& =\sum_{l=0}^{\infty}\left(\frac{h l+\alpha}{k}\right)_{m+n+J} \frac{\left(-p x^{h}\right)^{l}}{l !} \sum_{j=0}^{\infty} \frac{\left(p x^{h}\right)^{j}}{j !} \\
& =\exp \left(p x^{h}\right) \sum_{l=0}^{\infty}\left(\frac{h l+\alpha}{k}\right)_{m+n+J} \frac{\left(-p x^{h}\right)^{l}}{l !}
\end{aligned}
$$

and substituting this expression in (14), and applying the binomial expansion to sum the resulting $n$-series, we finally obtain

$$
\begin{aligned}
\Omega\left(u_{1}, \ldots, u_{r}\right)= & k^{m} \exp \left(p x^{h}\right) \Delta_{r}^{-m-\alpha / k} \\
& \cdot \sum_{l, j_{1}, \ldots, j_{r}=0}^{\infty}\left(\frac{h l+\alpha}{k}\right)_{m+J}\left(\frac{1}{l !}\right) \Lambda\left(j_{1}, \ldots, j_{r}\right)\left(-\frac{p x^{h}}{\Delta_{r}^{h / k}}\right)^{l} \\
& \cdot \prod_{i=1}^{r}\left\{\frac{\left[\left(-u_{i} / \Delta_{r}\right)^{m_{l}} y_{i}\right]^{j_{i}}}{j_{i} !}\right\}, \quad k \neq 0,
\end{aligned}
$$

where $\Delta_{r}$ and $J$ are given by (8) and (11), respectively, and the inequality in (10) is assumed to hold.

The right-hand sides of (9) and (15) are essentially the same. This evidently completes the proof of our theorem under the hypothesis that the various interchanges of the order of summation are permissible by absolute convergence of the series involved. Thus, in general, our theorem holds true whenever each member of (9) has a meaning.

REMARK. Our method of derivation can be applied mutatis mutandis in order to prove the following generalization of the multilinear generating function (9):

$$
\begin{aligned}
& \sum_{n_{1}, \ldots, n_{r}=0}^{\infty}\left(m+n_{1}+\cdots+n_{r}\right) ! \mathscr{F}_{m+n_{1}+\cdots+n_{r}}^{(\alpha)}(x, h, p, k) \\
& \mathscr{H}\left[n_{1}, \ldots, n_{r} ; y_{1}, \ldots, y_{r}\right] \frac{\left(u_{1} / k\right)^{n_{1}}}{n_{1} !} \cdots \frac{\left(u_{r} / k\right)^{n_{r}}}{n_{r} !} \\
= & k^{m} \exp \left(p x^{h}\right) \Delta_{r}^{-m-\alpha / k} \sum_{n, n_{1}, \ldots, n_{r}=0}^{\infty}\left(\frac{h n+\alpha}{k}\right)_{m+m_{1} n_{1}+\cdots+m_{r} n_{r}} \\
& \frac{\xi_{n}}{n !} \Delta\left(n_{1}, \ldots, n_{r}\right)\left(-\frac{p x^{h}}{\Delta_{r}^{h / k}}\right)^{n} \prod_{i=1}^{r}\left\{\frac{\left[\left(-u_{i} / \Delta_{r}\right)^{m_{2}} y_{i}\right]^{n_{i}}}{n_{i} !}\right\}, \quad k \neq 0, \quad k
\end{aligned}
$$


where, in terms of the bounded sequence $\left\{\xi_{n}\right\}$ of arbitrary complex numbers,

$$
\mathscr{F}_{n}^{(\alpha)}(x, h, p, k)=\frac{k^{n}}{n !} \sum_{j=0}^{\infty} \frac{\left(p x^{h}\right)^{j}}{j !} \sum_{l=0}^{j}(-1)^{l}\left(\begin{array}{l}
j \\
l
\end{array}\right) \xi_{l}\left(\frac{h l+\alpha}{k}\right)_{n},
$$

which obviously reduces to the Srivastava-Singhal equation (5) when $\xi_{l}=1, l \geq 0$.

3. Applications. By assigning suitable special values to the arbitrary coefficients $\Lambda\left(j_{1}, \ldots, j_{r}\right)$, the multiple sum in (7) can indeed be expressed in terms of the generalized Lauricella hypergeometric function of $r$ variables [11, p. 454]. Thus, following the various notations and conventions explained fairly fully by Srivastava and Daoust ([11, p. 545 et seq.]; see also [12]), we obtain from our theorem the multivariable hypergeometric generating function:

$$
\begin{aligned}
& \sum_{n_{1}, \ldots, n_{r}=0}^{\infty}\left(m+n_{1}+\cdots+n_{r}\right) ! G_{m+n_{1}+\cdots+n_{r}}^{(\alpha)}(x, h, p, k) \\
& \begin{array}{ccc}
\cdot F^{A}: 1+B^{\prime} ; \cdots ; 1+B^{(r)} & \left(\left[(a): \theta^{\prime}, \ldots, \theta^{(r)}\right]:\left[-n_{1}: m_{1}\right],\right. & {\left[\left(b^{\prime}\right): \phi^{\prime}\right] ; \cdots ;} \\
C: \quad D^{\prime} ; \cdots ; D^{(r)} & {\left[(c): \psi^{\prime}, \ldots, \psi^{(r)}\right]:} & {\left[\left(d^{\prime}\right): \delta^{\prime}\right] ; \cdots ;}
\end{array} \\
& \begin{aligned}
{\left[-n_{r}: m_{r}\right], } & {\left.\left[\left(b^{(r)}\right): \phi^{(r)}\right] ; y_{1}, \ldots, y_{r}\right)\left(\frac{u_{1}}{k}\right)^{n_{1}} \ldots\left(\frac{u_{r}}{k}\right)^{n_{r}} } \\
& {\left[\left(d^{(r)}\right): \delta^{(r)}\right] ; }
\end{aligned}
\end{aligned}
$$

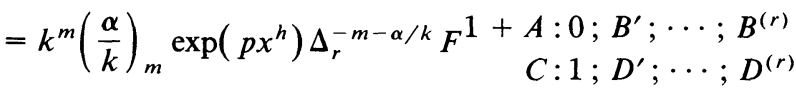

$$
\begin{aligned}
& \begin{array}{r}
{\left[m+\alpha / k: h / k, m_{1}, \ldots, m_{r}\right],\left[(a): 0, \theta^{\prime}, \ldots, \theta^{(r)}\right]:-} \\
{\left[(c): 0, \psi^{\prime}, \ldots, \psi^{(r)}\right]:[\alpha / k: h / k] ;}
\end{array} \\
& \begin{array}{l}
\left.\left[\left(b^{\prime}\right): \phi^{\prime}\right] ; \cdots ;\left[\left(b^{(r)}\right): \phi^{(r)}\right] ; \Xi_{0}, \Xi_{1}, \ldots, \Xi_{r}\right), \quad k \neq 0, \\
{\left[\left(d^{\prime}\right): \delta^{\prime}\right] ; \cdots ;\left[\left(d^{(r)}\right): \delta^{(r)}\right] ;}
\end{array}
\end{aligned}
$$

where $h / k>0, \Delta_{r}$ is given by (8), and

$$
\Xi_{0}=-\frac{p x^{h}}{\Delta_{r}^{h / k}}, \quad \Xi_{i}=y_{i}\left(-\frac{u_{i}}{\Delta_{r}}\right)^{m_{\iota}}, \quad i=1, \ldots, r .
$$

Next we set $A=C=0$ in (18) and, for convenience, let each of the positive coefficients $\phi_{j}^{(i)}, j=1, \ldots, B^{(i)} ; \delta_{j}^{(i)}, j=1, \ldots, D^{(i)}(i=1, \ldots, r)$ equal 1. Denoting the array of parameters

$$
\left(-n_{i}+j-1\right) / m_{i}, \quad j=1, \ldots, m_{i},
$$


by $\Delta\left(m_{i} ;-n_{i}\right), i=1, \ldots, r$, we thus find from (18) that

$$
\begin{aligned}
& \sum_{n_{1}, \ldots, n_{r}=0}^{\infty}\left(m+n_{1}+\cdots+n_{r}\right) ! G_{m+n_{1}+\cdots+n_{r}}^{(\alpha)}(x, h, p, k)
\end{aligned}
$$

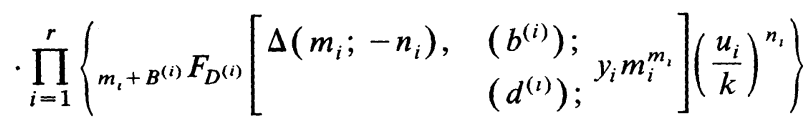

$$
\begin{aligned}
& =k^{m}\left(\frac{\alpha}{k}\right)_{m} \exp \left(p x^{h}\right) \Delta_{r}^{-m-\alpha / k}
\end{aligned}
$$

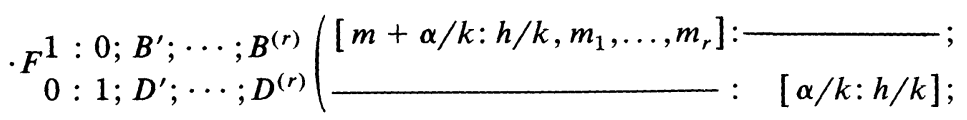

$$
\begin{aligned}
& \begin{array}{l}
\left.\left[\left(b^{\prime}\right): 1\right] ; \cdots ;\left[\left(b^{(r)}\right): 1\right] ; \Xi_{0}, \Xi_{1}, \ldots, \Xi_{r}\right), \quad k \neq 0, \\
{\left[\left(d^{\prime}\right): 1\right] ; \cdots ;\left[\left(d^{(r)}\right): 1\right] ;}
\end{array}
\end{aligned}
$$

where $h / k>0, \Delta_{r}$ is given by (8), and $\Xi_{0}, \Xi_{1}, \ldots, \Xi_{r}$ are defined by (19).

Obviously, this last formula (20) generates the product of $r$ generalized hypergeometric polynomials; it is a generalization of several known results due to Srivastava and Singhal [15].

For special values of the parameters, the Srivastava-Singhal polynomials $G_{n}^{(\alpha)}(x, h, p, k)$ can be reduced to the classical Hermite and Laguerre polynomials and their various generalizations studied in the literature ( $c f .[14$, p. 76]). Furthermore, the generalized hypergeometric polynomials occurring in (20) can be specialized to several important classes of hypergeometric polynomials including, for example, the classical Hermite polynomials and their such generalizations as those considered by Gould and Hopper [3, p. 58]

$$
\begin{aligned}
g_{n}^{m}(x, \lambda) & =\sum_{j=0}^{[n / m]} \frac{n !}{j !(n-m j) !} \lambda^{j} x^{n-m j} \\
& =x^{n}{ }_{m} F_{0}\left[\frac{\Delta(m ;-n) ;}{\left.\lambda\left(-\frac{m}{x}\right)^{m}\right]},\right.
\end{aligned}
$$

and by Brafman [1, p. 186]

$$
\begin{aligned}
\mathscr{B}_{n}^{m}\left[\alpha_{1}, \ldots, \alpha_{r} ; \beta_{1}, \ldots, \beta_{s}: x\right] & \\
& ={ }_{m+r} F_{s}\left[\begin{array}{ll}
\Delta(m ;-n), & \alpha_{1}, \ldots, \alpha_{r} ; x \\
& \beta_{1}, \ldots, \beta_{s} ;
\end{array}\right],
\end{aligned}
$$

where, as in (20), $\Delta(m ;-n)$ abbreviates the array of $m$ parameters

$$
(-n+j-1) / m, \quad j=1, \ldots, m,
$$


$m$ being an arbitrary positive integer. The details involved in these derivations of known or new multilinear generating functions from (20) may be left as an exercise to the interested reader.

Yet another interesting application of our theorem would result when in (18) we set

$$
\left\{\begin{array}{l}
h=p=1, \quad A=B^{(i)}=C=D^{(i)}-1=0, \\
d_{1}^{(i)}=1+\beta_{i}, \quad \delta_{1}^{(i)}=s_{i}, \quad m_{i}=1, \quad i=1, \ldots, r,
\end{array}\right.
$$

replace $\alpha$ by $\alpha+1$, and $y_{i}$ by $y_{i}^{s_{i}}, i=1, \ldots, r$, and appeal to the relationship (6) and to the explicit representation (2). We thus obtain our desired multilinear generating function for the Konhauser biorthogonal polynomials in the form:

$$
\begin{aligned}
& \sum_{n_{1}, \ldots, n_{r}=0}^{\infty}\left(m+n_{1}+\cdots+n_{r}\right) ! Y_{m+n_{1}+\cdots+n_{r}}^{\alpha}(x ; k) \\
& \cdot \prod_{i=1}^{r}\left\{Z_{n_{i}}^{\beta_{i}}\left(y_{i} ; s_{i}\right) \frac{u_{i}^{n_{i}}}{\left(1+\beta_{i}\right)_{s_{i} n_{i}}}\right\} \\
& =\left(\frac{\alpha+1}{k}\right)_{m} \quad e^{x} \Delta_{r}^{-m-(\alpha+1) / k} \\
& \cdot F_{0: 1 ; \cdots ; 1}^{1: 0 ; \cdots ; 0}(\underline{[m+(\alpha+1) / k: 1 / k, 1, \ldots, 1]}: \\
& \overline{[(\alpha+1) / k: 1 / k]} ; \quad \overline{\left[1+\beta_{1}: s_{1}\right]} ; \cdots ; \\
& \left.\overline{\left[1+\beta_{r}: s_{r}\right]} ;-\frac{x}{\Delta_{r}^{1 / k}},-\frac{u_{1} y_{1}^{s_{1}}}{\Delta_{r}}, \ldots,-\frac{u_{r} y_{r}^{s_{r}}}{\Delta_{r}}\right),
\end{aligned}
$$

where, by definition,

$$
\text { (24) } \alpha>-1 ; \quad \beta_{i}>-1 ; \quad k, s_{i}=1,2,3, \ldots ; \quad \forall i \in\{1, \ldots, r\} \text {. }
$$

A seriously erroneous version of a special case of the multilinear generating function (23), when $s_{1}=\cdots=s_{r}=s$, was proven earlier by Patil and Thakare [6] who incidentally used a markedly different method. In fact, (23) with $k=s_{1}=\cdots=s_{r}=1$ is a well-known result (involving the classical Laguerre polynomials) due to Srivastava and Singhal [15, p. 1239, Eq. (5)].

Since $s_{1}, \ldots, s_{r}$ are, by definition, positive integers, the multilinear generating function (23) would follow also as an obvious special case of (20). 


\section{REFERENCES}

[1] F. Brafman, Some generating functions for Laguerre and Hermite polynomials, Canad. J. Math., 9 (1957), 180-187.

[2] L. Carlitz, A note on certain biorthogonal polynomials, Pacific J. Math., 24 (1968), 425-430.

[3] H. W. Gould and A. T. Hopper, Operational formulas connected with two generalizations of Hermite polynomials, Duke Math. J., 29 (1962), 51-63.

[4] J. D. E. Konhauser, Some properties of biorthogonal polynomials, J. Math. Anal. Appl., 11 (1965), 242-260.

[5] , Biorthogonal polynomials suggested by the Laguerre polynomials, Pacific J. Math., 21 (1967), 303-314.

[6] K. R. Patil and N. K. Thakare, Multilinear generating function for the Konhauser biorthogonal polynomial sets, SIAM J. Math. Anal., 9 (1978), 921-923.

[7] S. Preiser, An investigation of biorthogonal polynomials derivable from ordinary differential equations of the third order, J. Math. Anal. Appl., 4 (1962), 38-64.

[8] L. Spencer and U. Fano, Penetration and diffusion of X-rays. Calculation of spatial distribution by polynomial expansion, J. Res. Nat. Bur. Standards, 46 (1951), 446-461.

[9] H. M. Srivastava, Certain double integrals involving hypergeometric functions, Jñānābha Sect. A, 1 (1971), 1-10.

[10] _ Some biorthogonal polynomials suggested by the Laguerre polynomials, Pacific J. Math., 98 (1982), 235-250.

[11] H. M. Srivastava and M. C. Daoust, Certain generalized Neumann expansions associated with the Kampé de Fériet function, Nederl. Akad. Wetensch. Proc. Ser. A, 72 = Indag. Math., 31 (1969), 449-457.

[12] A note on the convergence of Kampé de Fériet's double hypergeometric series, Math. Nachr., 53 (1972), 151-159.

[13] H. M. Srivastava and J.-L. Lavoie, A certain method of obtaining bilateral generating functions, Nederl. Akad. Wetensch. Proc. Ser. A, 78 = Indag. Math., 37 (1975), 304-320.

[14] H. M. Srivastava and J. P. Singhal, A class of polynomials defined by generalized Rodrigues' formula, Ann. Mat. Pura Appl. (4), 90 (1971), 75-85.

[15] _ Some formulas involving the products of several Jacobi or Laguerre polynomials, Acad. Roy. Belg. Bull. Cl. Sci. (5), 58 (1972), 1238-1247.

Received December 22, 1982. Supported, in part, by NSERC (Canada) Grant A-7353.

UNIVERSITY OF VICTORIA

VICTORIA, BRITISH COLUMBIA V8W 2Y2

CANAda 



\section{PACIFIC JOURNAL OF MATHEMATICS EDITORS}

DONALD BABBITT (Managing Editor)
University of California
Los Angeles, CA 90024
CHARLES R. DePrima
California Institute of Technology
Pasadena, CA 91125
R. FinN
Stanford University
Stanford, CA 94305

HERMANN FLASCHKA

University of Arizona

Tucson, AZ 85721

RAMESH A. GANGOLLI

University of Washington

Seattle, WA 98195

ROBION KIRBY

University of California

Berkeley, CA 94720

C. C. MOore

University of California

Berkeley, CA 94720
Hugo RossI

University of Utah

Salt Lake City, UT 84112

H. SAMELSON

Stanford University

Stanford, CA 94305

HaRold STARK

University of California, San Diego

La Jolla, CA 92093

\section{ASSOCIATE EDITORS}
R. ARENS
E. F. BECKENBACH (1906-1982)
B. H. NEUMANN
F. WOLF
K. YoshidA

\section{SUPPORTING INSTITUTIONS}

UNIVERSITY OF ARIZONA

UNIVERSITY OF BRITISH COLUMBIA

CALIFORNIA INSTITUTE OF TECHNOLOGY

UNIVERSITY OF CALIFORNIA

MONTANA STATE UNIVERSITY

UNIVERSITY OF NEVADA, RENO

NEW MEXICO STATE UNIVERSITY

OREGON STATE UNIVERSITY
UNIVERSITY OF OREGON

UNIVERSITY OF SOUTHERN CALIFORNIA

STANFORD UNIVERSITY

UNIVERSITY OF HAWAII

UNIVERSITY OF TOKYO

UNIVERSITY OF UTAH

WASHINGTON STATE UNIVERSITY

UNIVERSITY OF WASHINGTON 


\section{Pacific Journal of Mathematics}

\section{Vol. 117, No. $1 \quad$ January, 1985}

Amos Altshuler and Leon Steinberg, The complete enumeration of the

4-polytopes and 3 -spheres with eight vertices $\ldots \ldots \ldots \ldots \ldots \ldots \ldots \ldots$

Michael James Beeson, The $6 \pi$ theorem about minimal surfaces . . . . . . . . 17

Jeffrey Lawrence Caruso and Stefan Waner, An approximation theorem

for equivariant loop spaces in the compact Lie case ...............27

Jo-Ann Deborah Cohen, Topologies on the quotient field of a Dedekind

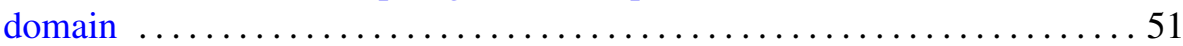

Szymon Dolecki, Gabriele H. Greco and Alois Andreas Lechicki,

Compactoid and compact filters .........................6 69

Roger William Hansell (Sr.), Generalized quotient maps that are

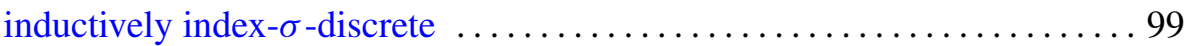

Gerhard Huisken, Capillary surfaces over obstacles $\ldots \ldots \ldots \ldots \ldots \ldots \ldots 121$

Jun Shung Hwang, A problem on continuous and periodic functions . . . . 143

Ronald Fred Levy and Michael David Rice, The extension of equi-uniformly continuous families of mappings $\ldots \ldots \ldots \ldots \ldots \ldots 149$

Kevin Mor McCrimmon, Derivations and Cayley derivations of generalized

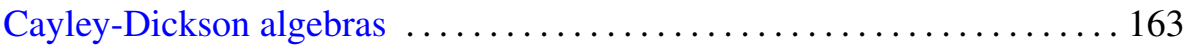

H. M. (Hari Mohan) Srivastava, A multilinear generating function for the Konhauser sets of biorthogonal polynomials suggested by the Laguerre

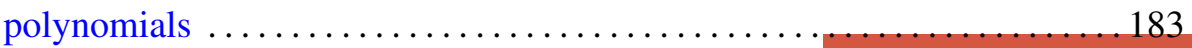

Zhu Jia Lu, Some maximum properties for a family of singular hyperbolic operators 